\title{
Experience of changing between signal and Bactec 9240 blood culture systems in a children's hospital
}

J Gray, M Brockwell, I Das

\begin{abstract}
Aim-To compare experience of positive blood cultures in successive years before and after changing from Signal (Unipath) to Bactec 9240 (Becton Dickinson) blood culture systems.

Methods-Analysis of data collected prospectively on 7967 Signal and 7062 Bactec blood culture sets.

Results-Significant growths occurred in $5.7 \%$ of Signal and $8.9 \%$ of Bactec cultures; $33.0 \%$ more significant isolates and $\mathbf{2 4 . 0 \%}$ more episodes of bacteraemia were detected in the second year, following introduction of the Bactec system. Inpatient hospital activity increased by $8.2 \%$ between the first and second years, although the numbers of blood cultures received actually fell by $11.4 \%$. There were striking increases in numbers of isolates of coagulase negative staphylococci $(47.7 \%)$ and Enterobacteriaceae $(56.8 \%)$ from Bactec cultures. Two anaerobic bacteraemias were detected in Signal blood cultures, whereas none was detected by the Bactec system, despite $12.1 \%$ of sets including an anaerobic bottle. Of significant positive cultures, $90.2 \%$ were detected within one day with the Bactec 9240, compared with only $50.0 \%$ of Signal cultures; $20.7 \%$ of significant positive Signal blood cultures were detected only on terminal subculture. Microorganisms that were not significant were isolated from $5.1 \%$ Signal and $3.8 \%$ Bactec cultures. Conclusions-Compared with the Signal system, the Bactec 9240 offers markedly more rapid and sensitive detection of bacteraemia, together with a lower rate of non-significant isolates. However, using a single PEDS PLUS/F bottle the few episodes of anaerobic bacteraemia that occur in children are likely to be missed.

(f Clin Pathol 1998;51:302-305)
\end{abstract}

Keywords: blood culture systems; bacteraemia

Department of

Microbiology,

Birmingham

Children's Hospital,

Ladywood,

Birmingham B16 8ET,

UK

J Gray

M Brockwell

I Das

Correspondence to:

Dr Gray.

Accepted for publication 29 January 1998 a state of the requires considerable ongoing additional financial outlay. It is therefore important to determine the benefits that are realisable in return for the increased cost.

There have been several studies comparing different blood culture systems. ${ }^{13-6}$ However, there are limited data comparing continuous monitoring systems with the manual systems that are still relatively widely used, especially by smaller laboratories. Our laboratory recently introduced the Bactec 9240 automated blood culture system (Becton Dickinson UK) to replace our previous manual Signal system (Unipath, Basingstoke, UK). In this paper we review our experience of detecting bacteraemia in the 12 month periods before and after this change.

\section{Methods}

SETTING

Birmingham Children's Hospital is a 220 bed paediatric teaching hospital and tertiary referral centre in the English Midlands. The work of the hospital includes provision of general paediatric services to the children of Birmingham, as well as various regional and supraregional services.

\section{BLOOD CULTURE SYSTEMS}

The Signal blood culture system (Unipath) was replaced by the Bactec 9240 system (Becton Dickinson UK) on September 181996.

Inoculated Signal blood culture bottles were incubated at $37^{\circ} \mathrm{C}$ for seven days (on an orbital shaker for the first 48 hours). The bottles were inspected several times a day, and those which signalled were sampled. Terminal subculture was carried out on all bottles that had not signalled. In addition, cultures where the request form indicated possible infection with Neisseria meningitidis or Haemophilus influenzae were routinely subcultured after 24 and 48 hours.

With the Bactec 9240, the PEDS PLUS/F bottle alone was used routinely. In addition, we recommended that an ANAEROBIC/F bottle should also be inoculated where children had underlying disease that might predispose to anaerobic infection, or where anaerobic infection was otherwise suspected clinically. For the purposes of analysis, a set of Bactec blood culture bottles was defined as any single bottle or combination of bottles inoculated with the same blood sample. The volume of blood added was not routinely measured; however, periodic audits have shown no evidence of a recent change in the pattern of inoculation of blood culture bottles. All cultures were incubated for seven days and discarded without terminal subculture.

With both systems, cultures becoming positive between 09.00 and 24.00 hours were sampled as soon as practicably possible. Investiga- 
tion of any positive prompts outside these hours was deferred until the next day.

Identification and antimicrobial susceptibility testing of isolates was performed using standard methods.

Prospective microbiological and clinical data on all positive blood cultures between $18 \mathrm{Sep}$ tember 1995 and 17 September 1997 were recorded and subsequently analysed using the EPI INFO 5 database and statistical package (Centers for Disease Control, Atlanta, Georgia, USA). All patients with positive blood cultures were assessed by a medical microbiologist. Bacteraemia was defined as detection of growth of microorganisms in one or more blood culture bottles in the presence of other laboratory or clinical evidence of infection. Any other positive blood cultures obtained before a response to treatment occurred were considered to represent the same episode. Statistical analysis was performed by the $\chi^{2}$ test with Yates correction where appropriate.

\section{Results}

HOSPITAL ACTIVITY AND NUMBER OF BLOOD CULTURES RECEIVED

We received 7967 Signal blood cultures in the year ending August 17 1996, compared with 7062 sets of Bactec blood cultures in the following year. These comprised 5644 single PEDS PLUS/F bottles, 642 sets of PEDS PLUS/F and ANAEROBIC/F bottles, and 134 ANAEROBIC/F bottles only. Although the numbers of blood cultures received decreased by $11.4 \%$ between the first and second years, numbers of inpatient finished consultant episodes increased by $8.2 \%$ from 18908 to 20451 .

POSITIVE BLOOD CULTURES

Growth was detected in 864 (10.8\%) Signal blood cultures, yielding a total of 700 different microorganisms; 458 cultures $(5.7 \%)$ were considered to be significant, yielding 267 different microorganisms, and representing 246 episodes of bacteraemia. Sixteen episodes $(6.5 \%)$ were polymicrobial. Growth was detected in 893 sets of Bactec blood cultures $(12.6 \%)$, yielding 668 different microorganisms; 355 isolates obtained from 627 sets $(8.9 \%)$ were considered significant, representing 305 episodes of bacteraemia. Thirty nine episodes $(12.8 \%)$ were polymicrobial. Overall, $33.0 \%$ more significant isolates and $24.0 \%$ more episodes of bacteraemia were detected in the year following introduction of the Bactec system.

Significant isolates detected by the two systems are shown in table 1 . Notable changes following the introduction of the Bactec 9240 include increases of $31.4 \%$ and $47.7 \%$ in the numbers of isolates of Staphylococcus aureus and coagulase negative staphylococci, and a $56.8 \%$ increase in the number of enterobacteriaceae. There were 10 isolates of $N$ meningitidis, compared with five in the previous year. Two episodes of clostridium bacteraemia were detected with the Signal system, whereas no anaerobic bacteria were isolated in Bactec cultures.
Table 1 Significant blood culture isolates detected in successive one year periods before and after replacement of the Signal blood culture system with the Bactec 9240

\begin{tabular}{|c|c|c|}
\hline \multirow[b]{2}{*}{ Species } & \multicolumn{2}{|c|}{ No (\%) of isolates } \\
\hline & Signal & Bactec \\
\hline Coagulase negative staphylococci & $86(32.2)$ & $127(35.8)$ \\
\hline$S$ aureus & $35(13.1)$ & $46(13.0)$ \\
\hline Enterococcus spp & $23(8.6)$ & $21(5.9)$ \\
\hline Str pneumoniae & $10(3.7)$ & $10(2.8)$ \\
\hline$\beta$ Haemolytic streptococci & $6(2.2)$ & $10(2.8)$ \\
\hline Viridans streptococci & $14(5.2)$ & $17(4.8)$ \\
\hline Bacillus spp & $6(2.2)$ & $7(2.0)$ \\
\hline Other Gram positive & $6(2.2)$ & $10(2.8)$ \\
\hline Total Gram positive & $186(69.7)$ & $248(69.9)$ \\
\hline E coli & $13(4.9)$ & $18(5.1)$ \\
\hline Klebsiella spp & $10(3.7)$ & $19(5.4)$ \\
\hline Enterobacter spp & $8(3.0)$ & $12(3.4)$ \\
\hline Other Enterobacteriaceae & $6(2.2)$ & $9(2.5)$ \\
\hline Ps aeruginosa & $8(3.0)$ & $8(2.3)$ \\
\hline Pseudomonas spp & $8(3.0)$ & $4(1.1)$ \\
\hline Acinetobacter spp & $7(2.6)$ & $6(1.7)$ \\
\hline$N$ meningitidis & $5(1.9)$ & $10(2.8)$ \\
\hline H influenzae & $4(1.5)$ & $3(0.8)$ \\
\hline Other Gram negative & $4(1.5)$ & $9(2.5)$ \\
\hline Total Gram negative & $73(27.3)$ & $98(27.6)$ \\
\hline Clostridium spp & $2(0.7)$ & - \\
\hline Total anaerobes & $2(0.7)$ & - \\
\hline Candida spp & $6(2.2)$ & $9(2.5)$ \\
\hline Total fungi & $6(2.2)$ & $9(2.5)$ \\
\hline Total isolates & 267 & 355 \\
\hline
\end{tabular}

Table 2 Non-significant blood culture isolates detected in successive one year periods before and after replacement of the Signal blood culture system with the Bactec 9240

\begin{tabular}{lcc}
\hline \multicolumn{3}{c}{$N o(\%)$ of isolates } \\
\cline { 2 - 3 } Species & Signal & Bactec \\
\hline Coagulase negative & $274(63.3)$ & $213(68.1)$ \\
$\begin{array}{l}\text { Staphylococci } \\
\text { Saureus }\end{array}$ & $6(1.4)$ & $1(0.3)$ \\
"Diphtheroids" & $62(14.3)$ & $23(7.3)$ \\
Micrococcus spp & $4(0.9)$ & $6(1.9)$ \\
Viridans streptococci & $46(10.6)$ & $43(13.7)$ \\
Bacillus spp & $8(1.8)$ & $4(1.3)$ \\
Other Gram positive & $9(2.1)$ & $7(2.2)$ \\
Total Gram positive & $\mathbf{4 0 9}(\mathbf{9 4 . 5})$ & $\mathbf{2 9 7}(\mathbf{9 4 . 9 )}$ \\
Pseudomonas spp & $17(3.9)$ & $2(0.6)$ \\
Acinetobacter spp & $4(0.9)$ & $1(0.3)$ \\
Neisseria spp & $2(0.5)$ & $9(2.9)$ \\
Total Gram negative & $\mathbf{2 3}(\mathbf{5 . 3 )}$ & $\mathbf{1 2}(\mathbf{3 . 8})$ \\
Total fungi & $\mathbf{1}(\mathbf{0 . 2})$ & $\mathbf{1}(\mathbf{0 . 3 )}$ \\
Total isolates & $\mathbf{4 3 3}$ & $\mathbf{3 1 3}$ \\
\hline
\end{tabular}

Four hundred and six Signal blood cultures $(5.1 \%)$ contained microorganisms that were not significant, compared with 266 sets of Bactec cultures (3.8\%); 27.7\% more isolates that were not considered to be significant were obtained with the Signal system (table 2).

The times taken for detection of significant and non-significant growths in blood cultures with the two systems are shown in table 3. Over $90 \%$ of significant positive blood cultures were detected with the Bactec 9240 after one day, whereas over $20 \%$ of positive Signal blood cultures were only detected in the terminal subculture.

PATIENT CHARACTERISTICS

Of the patients with significant positive Signal and Bactec blood cultures, $89.4 \%$ and $88.5 \%$ respectively had significant underlying disease $(\mathrm{p}=0.7)$. The proportions of bacteraemias detected in different specialties and age groups were similar during the two periods of study (data not shown).

Fifty three patients with bacteraemia $(21.5 \%)$ were receiving antibiotic treatment at 
Table 3 Comparison of detection times for significant and non-significant blood cultures in successive one year periods before and after replacement of the Signal blood culture system with the Bactec 9240

\begin{tabular}{lccccc}
\hline & \multicolumn{2}{l}{ No (\%) of blood cultures containing isolates that were: } \\
\cline { 2 - 3 } \cline { 5 - 6 } $\begin{array}{l}\text { No of } \\
\text { days }\end{array}$ & Significant & & & \multicolumn{2}{l}{ Non-significant } \\
\cline { 2 - 3 } \cline { 5 - 6 } & & Bactec & & Signal & Bactec \\
\hline$<1$ & $23(5.0)$ & $182(29.0)$ & & $3(0.7)$ & $43(16.2)$ \\
1 & $206(45.0)$ & $384(61.2)$ & & $105(25.9)$ & $153(57.5)$ \\
2 & $129(28.2)$ & $42(6.7)$ & & $158(38.9)$ & $38(14.3)$ \\
3 & $23(5.0)$ & $9(1.4)$ & & $26(6.4)$ & $10(3.8)$ \\
4 & $14(3.1)$ & $6(1.0)$ & & $12(3.0)$ & $10(3.8)$ \\
5 & $6(1.3)$ & $2(0.3)$ & & $9(2.2)$ & $4(1.5)$ \\
6 & $8(1.7)$ & - & & $9(2.2)$ & $3(1.1)$ \\
7 & $49(10.7)$ & $2(0.3)$ & & $84(20.7)$ & $5(1.9)$ \\
\hline
\end{tabular}

the time of detection of their bacteraemias using the Signal system, compared with 79 $(25.9 \%)$ with the Bactec $9240(\mathrm{p}=0.8)$.

\section{Discussion}

Patients with bacteraemia who receive appropriate antimicrobial treatment promptly have an improved outcome. ${ }^{8}$ The importance of a rapid and sensitive blood culture method is underlined by our experience that $38.7 \%$ of septicaemic episodes were either untreated or inappropriately treated until the results of blood cultures became available.

In the year following introduction of the Bactec system, $33.0 \%$ more significant blood culture isolates and $24.0 \%$ more episodes of bacteraemia were detected than in the previous year. These differences were seen across all patient groups, and occurred against a background of a decrease of $11.4 \%$ in the number of blood culture sets received. Although hospital inpatient activity increased by $8.2 \%$ over the same period, much of the additional workload arose from patients who were unlikely to become septicaemic. It is therefore likely that the increased number of blood culture isolates obtained with the Bactec system largely reflects improved detection rather than a greater incidence of bacteraemia.

One of the purposes of resins incorporated into Bactec media is to neutralise the effects of antibiotics. ${ }^{2}$ However, we found no significant difference between the two systems in the proportions of patients with bacteraemia who were receiving antibiotic treatment. Possibly the dilutional effect of adding small blood volumes from children to the relatively large volume of Signal blood culture medium helps to negate the effect of antibiotics.

Compared with other blood culture systems, Bactec systems have been shown to be especially effective in detecting staphylococci, ${ }^{3-6}$ while the Bactec 9240 has been reported to be more sensitive than earlier generation Bactec systems. ${ }^{1}$ We also found large increases in the numbers of episodes of bacteraemia with $S$ aureus, and especially coagulase negative staphylococci, following the introduction of the Bactec 9240. As in many other specialist hospitals, ${ }^{9}{ }^{10}$ coagulase negative staphylococci are the most common cause of significant bacteraemia in our hospital. Improved detection of Enterobacteriaceae with
Bactec systems has been reported less consistently ${ }^{13-6}$; however, in our hands $56.8 \%$ more episodes of bacteraemia were detected. Although the numbers of episodes were small, we also detected more cases of meningococcaemia and fungaemia with the Bactec 9240.

Anaerobic bacteraemia is uncommon in children ${ }^{11}$ and no episodes were detected with the Bactec system, despite an anaerobic bottle being included in $12.1 \%$ of the blood culture sets. The two episodes of clostridium bacteraemia detected with the Signal system were in children with neutropenia secondary to anticancer chemotherapy. Our protocol does not recommend anaerobic blood culture in these patients, although clostridia are well recognised as uncommon but potentially serious pathogens in this setting. ${ }^{12}{ }^{13}$ One of the children did not receive antibiotic treatment until the blood culture results were available.

There may be little advantage in responding immediately to positive blood cultures detected by continuous monitoring systems. ${ }^{14}$ We react to positives detected by the Bactec 9240 in the same way as we did with the Signal system, and in particular do not immediately investigate cultures that become positive after midnight. Thus number of days (rather than hours) to detection of positives is the most valid and clinically relevant measure in comparing the two systems. Of significant positive Signal blood cultures, $78.2 \%$ were detected by the second day, compared with $80.6 \%$ in a previous study. ${ }^{15}$ By comparison, over $90 \%$ of significant Bactec cultures were positive within one day.

Blood culture isolates that are not significant can lead to considerable unnecessary costs in terms of additional testing, unnecessary antibiotic treatment, and increased length of hospital stay. ${ }^{16}$ Non-significant isolates were obtained from $5.1 \%$ of Signal cultures, compared with $6.9 \%$ in a previous study from our hospital. ${ }^{15}$ The lower contamination rate in the current study may stem from the fact that a large proportion of the blood cultures that we receive are now collected through intravascular devices, which are easier to sample. The contamination rate with the Bactec system was only $3.8 \%$, which is in accordance with previously reported rates of $1.6 \%$ to $3.8 \% .{ }^{15}$ The lower contamination rate with the Bactec system is partly explained by a decrease in the numbers of "anaerobic diphtheroids," which were rarely recovered from PEDS PLUS/F bottles. However, there were also large decreases in the numbers of staphylococci and pseudomonads, suggesting that contaminants can be introduced when Signal blood culture bottles are manipulated in the laboratory. It is well recognised that these species occur as contaminants during laboratory manipulation of blood cultures. ${ }^{17}$

Compared with the Signal system, the Bactec 9240 appears to offer markedly more rapid and sensitive detection of bacteraemia in both general and specialist paediatric patients, while also being associated with a significantly lower contamination rate. The only concern is that by using the PEDS PLUS/F bottle alone the 
few episodes of anaerobic bacteraemia that occur in children are likely to be missed.

1 Nolte FS, Williams JM, Jerris RC, et al. Multicenter clinical evaluation of a continuous monitoring blood culture system using fluorescent-sensor technology (BACTEC 9240). $\mathcal{F}$ Clin Microbiol 1993;31:552-7.

2 Crepin O, Roussel-Delvallez M, Martin GR, et al. Effectiveness of resins in removing antibiotics from blood cultures f Clin Microbiol 1993;31:734-5.

3 Morello JA, Matushek SM, Dunne WM, et al. Performance of a BACTEC nonradiometric medium for pediatric blood cultures. $\mathcal{F}$ Clin Microbiol 1991;29:359-62

4 Rimmer K, Cabot M. Comparison of Bactec NR-660 and Signal systems. F Clin Pathol 1988;41:676-8.

5 Smith JA, Bryce EA, Ngui-Yen JH, et al. Comparison of BACTEC 9240 and BacT/Alert blood culture systems in an adult hospital. $\mathcal{F}$ Clin Microbiol 1995;33:1905-8.

6 Zaidi AK, Mirrett S, McDonald JC, et al. Controlled Zaidi AK, Mirrett S, McDonald JC, et al. Controlled
comparison of bioMerieux VITAL and BACTEC NR-660 comparison of bioMerieux VITAL and BACTEC NR-660
systems for detection of bacteremia and fungemia in pedisystems for detection of bacteremia and fungemi
atric patients. $\mathcal{F}$ Clin Microbiol 1997;35:2007-12.

7 Collee JG, Duguid JP, Fraser AG, et al, eds. Mackie and Collee JG, Duguid JP, Fraser AG, et al, eds. Mackie and
McCartney. Practical medical microbiology, 13th ed. Edinburgh: Churchill Livingstone, 1989.

8 Weinstein MP, Murphy JR, Reller LB, et al. The clinical significance of positive blood cultures: a comprehensive analysis of 500 episodes of bacteremia and fungemia in adults. II. Clinical observations, with special reference to
factors influencing prognosis. Rev Infect Dis 1983;5:54-70. 9 Neu HC, Fracaro M, Bopp H, et al. Bacteraemia-a New York perception. $\mathcal{F}$ Antimicrob Chemother 1990;25(suppl York percep

10 Váquez F, Mendoza MC, Villar MH, et al. Survey of bacteraemia in a Spanish hospital over a decade (1981-1990). $\mathcal{F}$ Hosp Infect 1994;26:111-21.

11 Zaidi AK, Knaut AL, Mirrett S, et al. Value of routine anaerobic blood cultures for pediatric patients. $\mathcal{F}$ Pediatr 1995;127:263-8.

12 Clostridium septicum and neutropenic enterocolitis. Lancet 1987;ii: 608

13 Gray JW, Pedler SJ, Craft AW, et al. Changing causes of septicaemia in paediatric oncology patients: effect of imipenem use. Eur 7 Pediatr 1994;153:84-9.

14 Murdoch DA, Koerner RJ, Speirs GE, et al. Do blood cultures need continuous monitoring so that clinical action can be taken outside normal working hours? f Clin Pathol 1995; 48:1067-8.

15 Fox H, Healing DE, George RH. Evaluation of use of Signal system of blood culture in paediatrics. $f$ Clin Pathol system of blood

16 Bates DW, Goldman L, Lee TH. Contaminant blood cultures and resource utilization. The true consequences of false-positive results. $\mathcal{F} A M A$ 1991;265:365-9.

17 MacGregor RR, Beaty HN. Evaluation of positive blood cultures. Guidelines for early differentiation of contaminated from valid positive cultures. Arch Intern Med 1972;130:84-7. 